\title{
TECHNIQUES IN OPTICAL DATA PROCESSING AND COHERENT OPTICS
}

\author{
A. Vander Lugt and E. N. Leith \\ Institute of Science and Technology \\ The University of Michigan, Ann Arbor, Mich.
}

\section{Introduction}

Since analyses of coherent optical data processing systems have been extensively reported, ${ }^{1-8}$ we present only a brief account of their operation in this paper. FIGURE 1 shows a typical coherent optical data processing system. A point source of monochromatic light, collimated by lens $L_{c}$, illuminates the input data. The specular amplitude transmittance* of the input data in plane $P_{1}$ is denoted by $f(x, y)$. An inherent property of coherent systems is that a spherical lens placed to the right of the plane $P_{1}$ will display the two-dimensional Fourier transform $F(p, q)$ of the input data; i.e.,

$$
F(p, q)=\int_{-\infty}^{\infty} \int_{f} f(x, y) e^{j(p x+q y)} d x d y
$$

where $\mathrm{p}$ and $\mathrm{q}$ are radian spatial frequencies. Thus, the light distribution in plane $P_{2}$ is the spatial frequency decomposition of the input data $f(x, y)$; it contains both the amplitude and the phase information contained in the spectrum of the data.

A spatial filter whose transmittance is $H(p, q)$ can be placed in plane $P_{2}$ to modify $F(p, q)$ in some prescribed fashion. Lens $L_{2}$ displays the Fourier transform of the modified spectral distribution $F(p, q) H(p, q)$ in plane $P_{3}$; i.e., if $r(x, y)$ represents the output light distribution, then

$$
r(x, y)=\frac{1}{4 \pi^{2}} \int_{-\infty}^{\infty} \int_{-\infty} F(p, q) H(p, q) e^{j(p x+q y)} d p d q .
$$

By using the convolution theorem, we can write Equation 2 in terms of the impulse response $h(x, y)$ of the filter as

$$
r(x, y)=\int_{-\infty}^{\infty} \int_{-\infty} f(u, v) h(x-u, y-v) d u d v,
$$

which is a two-dimensional convolution operation. Equations 2 and 3 show that the optical system can perform a general linear integral operation similar to those performed by linear time-invariant electronic systems, except that the operation is two-dimensional. Also, note that scanning of the input data is not necessary so that the input data are processed in parallel.

The optical processor can be easily converted to a multichannel one-dimen-

- Coherent optical systems are linear in light amplitudes; hence, throughout this paper all light distributions and transmittances of photographic film or other light modulators will be considered amplitude functions. 


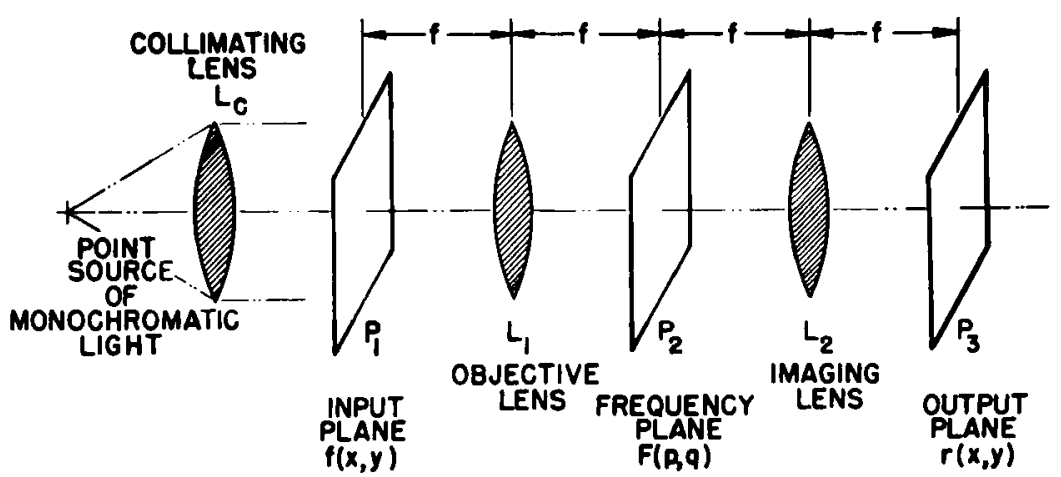

Figure 1. A typical two-dimensional coherent optical data-processor.

sional processor by replacing lenses $\mathrm{L}_{1}$ and $\mathrm{L}_{2}$ with cylindrical-spherical lens combinations. ${ }^{4}$ We denote the data for the $i$-th channel by $f(x, i \Delta y)$. The first cylindrical-spherical lens images the data in the $y$-direction while displaying its Fourier transform in the $\mathrm{x}$-direction. Thus, the light distribution in plane $\mathbf{P}_{\mathbf{2}}$ is

$$
F(p, i \Delta y)=\int_{-\infty}^{\infty} f(x, i \Delta y) e^{j p x} d x .
$$

We can now place a multichannel one-dimensional filter $H(p, i \Delta y)$ in plane $P_{2}$ to modify $F(p, i \Delta)$. This filter can have a different form for each channel, if desired. The second cylindrical-spherical lens then images $R(p, i \Delta y)=F(p$, $\mathrm{i} \Delta \mathrm{y}) \mathrm{H}(\mathrm{p}, \mathrm{i} \Delta \mathrm{y})$ in the $\mathrm{y}$-direction while displaying the Fourier transform in the $\mathrm{x}$-direction. The output is then

$$
r(x, i \Delta y)=\frac{1}{2 \pi} \int_{-\infty}^{\infty} F(p, i \Delta y) H(p, i \Delta y) e^{j p x} d p,
$$

or, by the convolution theorem,

$$
r(x, i \Delta y)=\int_{-\infty}^{\infty} f(u, i \Delta y) h(x-u, i \Delta y) d u,
$$

which is a multichannel one-dimensional convolution operation. If all channels of the input data are to be processed in the same way, the optical system shown in FIGURE 1 can be used directly, the only difference being that the spatial filter has uniform response in the y-direction.*

The form of the spatial filter function $H(p, q)$ will depend, of course, on the information we want extracted from the data. In this section we concern ourselves with the problem of detecting and recognizing certain patterns in two-dimensional data, or certain one-dimensional functions out of a large class of such functions. These problems can be analyzed within the framework of detecting a known sig-

- The optical system shown in FIgure 1 can also be used as a multichannel correlator by using the technique of multiplexed spatial filters. ${ }^{7}$ 

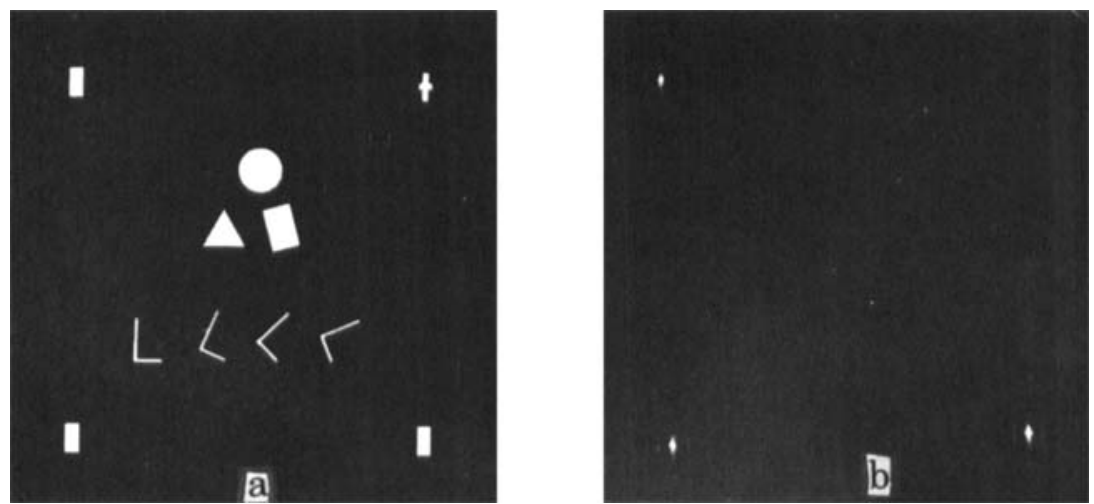

Figure 2. Detection of a rectangle. (a) A set of geometric shapes. (b) Output from the optical processor showing the detection of the small rectangles.

nal (with some unknown parameters) in a noisy background. If the signal is known, and if the signal and noise are additive, the filter that maximizes the ratio of peak-signal to mean-square noise is $\dagger^{9}$

$$
H(p, q)=\frac{C^{*}(p, q)}{|N(p, q)|^{2}}
$$

where $S(p, q)$ is the Fourier transform of the signal, $|N(p, q)|^{2}$ is the noise spectral density, $\mathrm{C}$ is a constant, and denotes conjugate. The filter function given by Equation 7 is usually called a "matched filter" because it is matched to both the phase and the amplitude of the spectrum of the signal. The filter is, of course, generally a complex-valued function and we must be able to record it on a suitable medium so that it can then modify $F(p, q)$.

Several interferometric optical systems for generating the appropriate filter functions on a recording media have been described elsewhere, ${ }^{10}$ and will not be described here. Suffice it to say that these techniques are well developed and that filters for several different signals can be recorded on a single filter so that the input data can be searched for several signals simultaneously. ${ }^{7}$

\section{Application to Pattern Recognition}

Although Equation 3 shows that a coherent optical system can perform a general integral operation, we will limit our attention to the specific application of pattern recognition. None of the examples shown are directly related to processing optical images in the medical and biological sciences, but they closely parallel problems that might be expected to arise in these sciences. Figure 2 shows a set of geometrical shapes. We constructed a filter matched to the spectrum of the small rectangle and the output of the system is shown in FIGURE $2 \mathrm{~b}$. Note that the three rectangles are detected simultaneously, showing that the optical system (by virtue of frequency-plane processing) does not have to scan the input data to determine the location of the signals. This feature results in large savings of processing time.

To detect signals having a random orientation, the filter can be rotated through $2 \pi$ radians, and the signal is detected when the filter is aligned with the signal.

$\dagger$ If the noise is also Gaussian, this filter is optimum in the sense of minimizing risk. 
Coupled with the nonscanning feature of the system, this capability results in even more savings of processing time.

FIGURE 3 shows another example of shape recognition using a slightly different technique. All of the shapes in FIGURE $3 \mathrm{a}$ were recorded on the filter to yield a multiple reference signal set. An unknown signal was then placed in the input and the location of the bright spot in the output served to identify the unknown signal.

In the first example, several filters were needed to recognize all the shapes; i.e., we time-shared the frequency domain. In the second example, we time-shared the space domain to process a large amount of data. By using multiplexed filters, we avoided time-sharing in either domain. Suppose we would wish to implement our optical system to identify a set of symbols where each symbol might take on one of nine different shapes. The set of nine shapes (arranged conveniently) is shown in FIGURE 4a. A multiplexed filter, shown in FIGURE $4 b$, was constructed to record this set of symbols and its impulse response is shown in FIGURE $4 c$. The separation between the nine symbols is large enough so that a nine-digit sequence, such as that shown in FIGURE 5, can be processed. We chose this sequence so the output will show how well each symbol correlates with itself, and also show how well the filter rejects the other symbols.
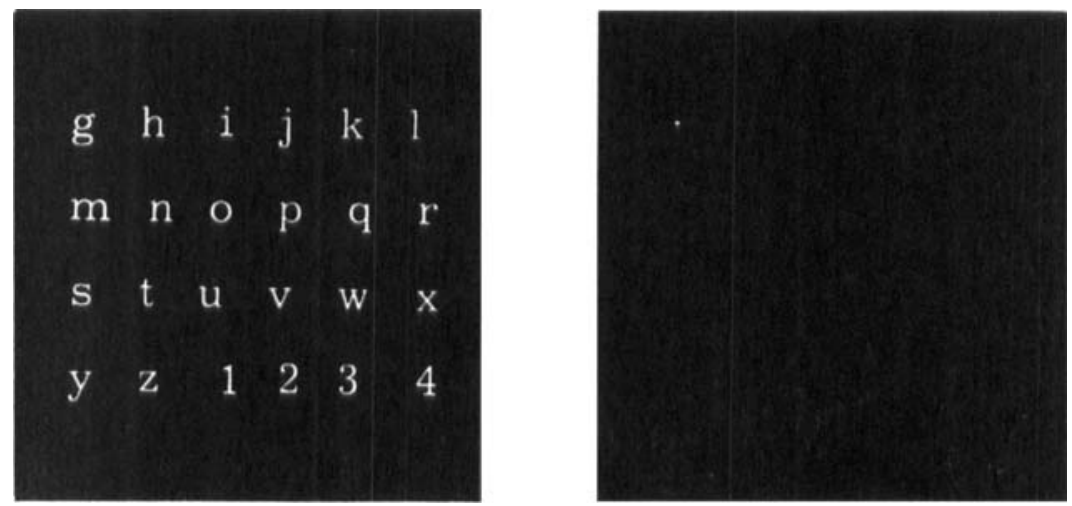

FIGURE 3. Detection of an alpha-numeric. (a) A set of characters used to construct the filter. (b) Detection of the character "g".
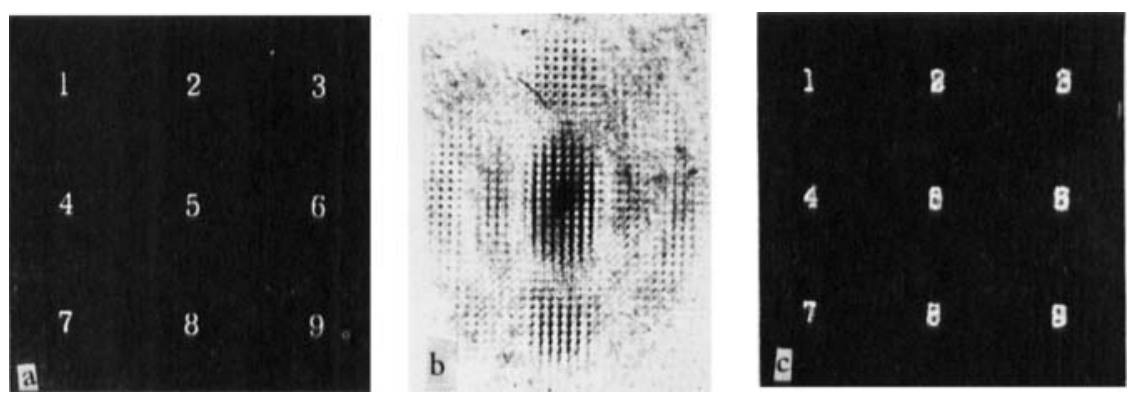

FIgURE 4. Example of multiplexed spatial filtering. (a) A set of nine numbers. (b) Multiplexed spatial filter. (c) Impulse response of the filter. 


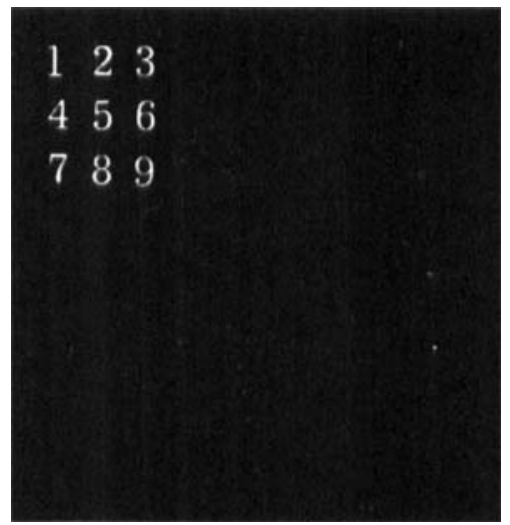

Figure 5. Input to the optical system.
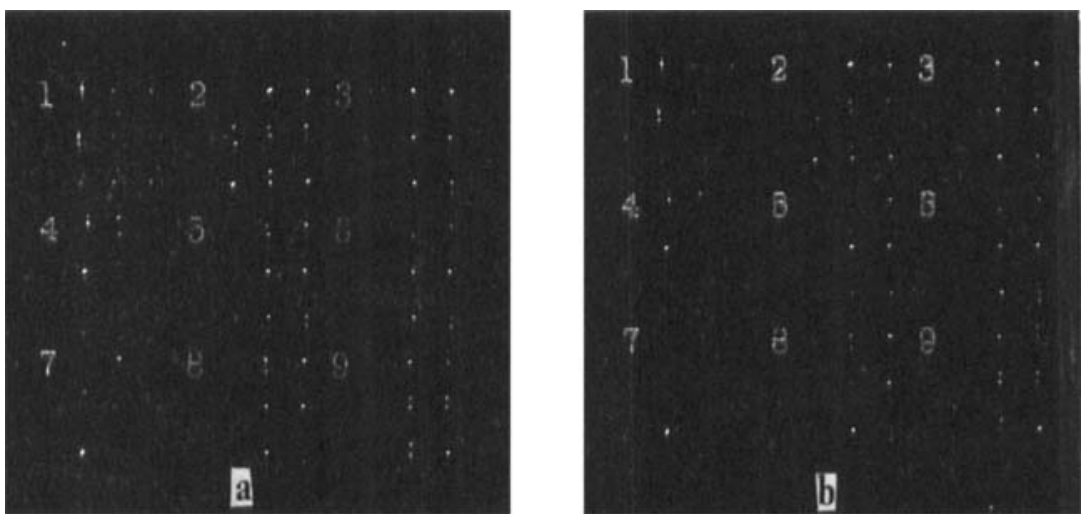

FIgure 6. Output showing multiple signal detection. (a) Output using a broadband filter. (b) Output using a high-pass filter.

Two filters were constructed, one with wideband frequency content and one with more emphasis on the high-frequency content. What we expect to gain from the latter filter is increased shape selectivity (or, alternatively, more noise rejection). However, we gain this selectivity at the expense of tolerance to variations in signal size, orientation, and quality.

The results are shown in FIGURE $6 a$ for the broadband filter and in FIGURE $6 \mathrm{~b}$ for the high-pass filter. To make the results somewhat clearer, we have included the impulse responses of the respective filters in the output by using a double exposure. Let us discuss FIGURE $6 \mathrm{a}$ in some detail so the results are clearly understood. First, we see the impulse response of the filter, which is similar to that shown in Figure 4c. To the right and below each symbol we see the output corresponding to the cross-correlation of that symbol with the input sequence that was shown in FIGURE 5. For example, to the right and below the symbol "4" in FIGURE $6 \mathrm{a}$ we see that the brightest spot of light occurs in the first column, second row. Referring to FIGURE 4 we see that this is the location of the symbol "4." A similar check with the other symbols shows that maximum correlation occurs at the point where that symbol occurs in the input. Also, within each 
subportion of the output. one can see how well the filter rejects the other (nonsignal) symbols.

It should be observed that the relative amount of light in the autocorrelation peak for each signal varies. This is because the original signals do not contain equal energies, and is most clearly illustrated in the output for the symbol " 1 ". If photodetectors were used to sense the correlation peaks, they could be biased properly to compensate for the inequalities of signal energy. The compensation could also be made when the filter is constructed.

From these examples, it should be apparent that coherent optical data processing has application to problems in the medical and biological sciences. Such problems as chromosome classification, virus detection, blood cell counting, the detection of aberrant blood cells, etc., should be prime candidates for solution by optical processing. Other applications are the rapid and accurate comparison of one-dimensional time functions such as electrocardiograms or electroencephalograms.

\section{Holography-Possible Application to Biological Situations}

Holography is currently a widely discussed technique in modern optics. While it encompasses only a narrow scope of coherent optics technology it has become increasingly useful as a result of numerous ramifications that have developed in the past few years. We will discuss here only those aspects of holography that are germane to the medical and biological sciences. Our discussion emphasizes three overlapping topics: holographic microscopy, holographic interferometry, and the construction and use of holographic lens elements.

Holography, a two-step imaging process introduced in 1948 by Gabor, ${ }^{10}$ may be defined as the process of recording, by photographic means, an arbitrary electromagnetic wave field so that both the phase and the amplitude of the field are preserved. The recorded wave field is preserved on the record until released. To release the wave, we need only illuminate the record, or hologram, with an appropriate light source, whereupon the released waves begin propagating in the same way as the original field was propagating when the hologram was made. An observer looking at the hologram sees the wave field as if it were indeed emanating from the original object, i.e., the observer sees what to all appearances is the original object, complete in full three-dimensional form and with all the normal parallax relations of real life. This dramatic realism has been responsible for the enormous current interest in holography.

The holographic process is shown in FIGURE 7. For a more detailed explanation, we refer the reader elsewhere ${ }^{11}$ and concentrate here on some possibly useful applications of this technique.

Gabor invented holography for the purpose of improving electron microscopy. El-Sum, Baez, ${ }^{12}$ and Kirkpatrick later investigated the possibility of making holograms with $x$-rays. Their efforts, had they been highly successful, would certainly have been revolutionary since there is much room for improved resolution in the areas of electron and x-ray microscopy. However, as their efforts met only limited success they are now primarily of historical interest.

Holography today has notable potential for microscopy in the visible part of the spectrum. By recording the output of a microscope as a hologram, we produce a record in which a three-dimensional specimen is completely preserved, and we may, by examining the hologram, bring into focus any section of the specimen that we wish. The hologram is thus equivalent to a series of photomicrographs made of successive planes of the specimen. 

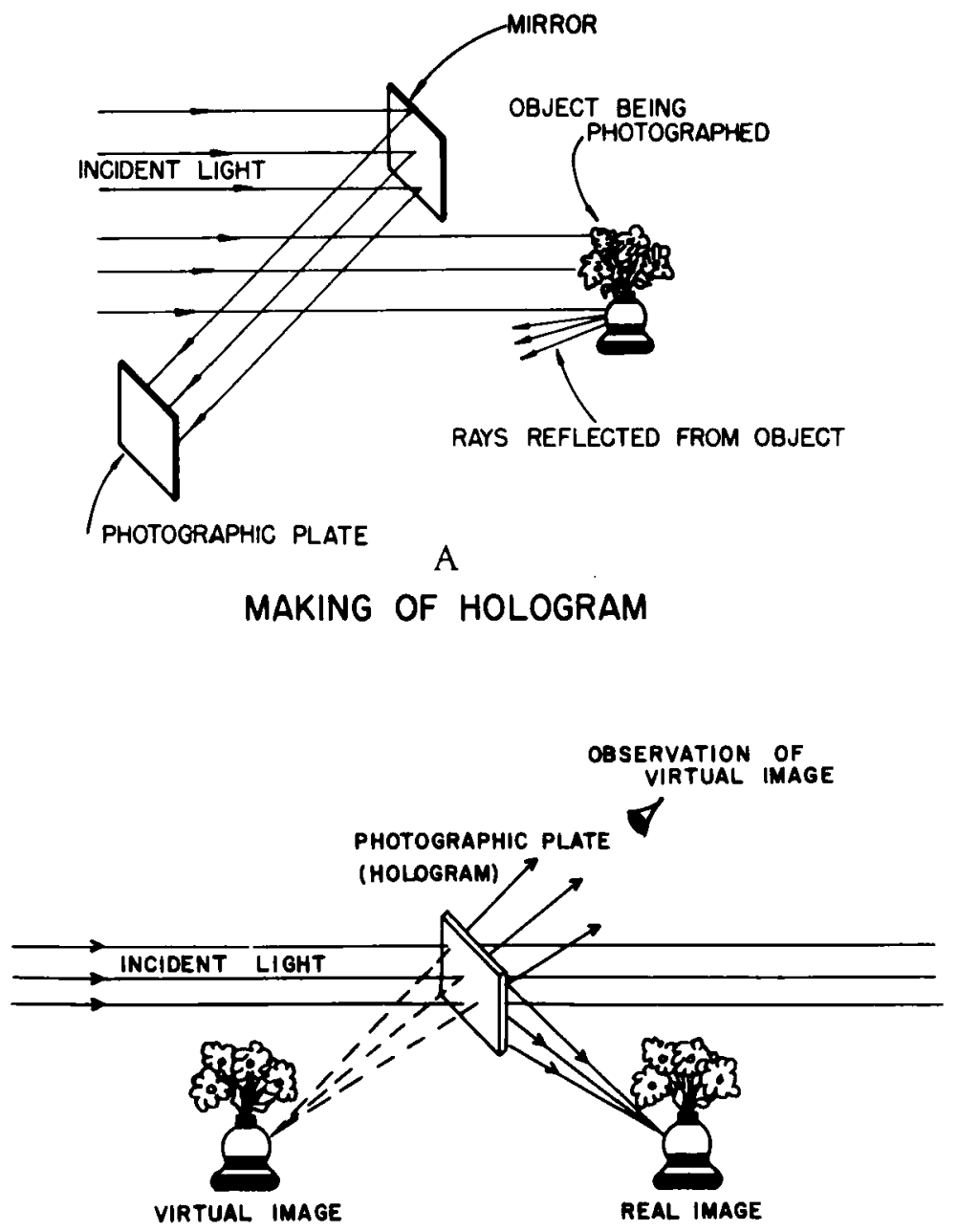

$\mathrm{B}$

\section{RECONSTRUCTION OF IMAGE}

Figure 7. The holographic process. (a) Forming the hologram. (b) Viewing the hologram. 
There are three basic methods for hologram microscopy, distinguished by the manner in which magnification is achieved. We may make a hologram of the output of a microscope, we may make a hologram without magnifying the object and then observe the holographic image with a microscope, or, finally, we may produce magnification without lenses by utilizing the image-forming properties of the hologram.

The third method is shown in FIGURE 8. The hologram is made and viewed using light of appropriate divergence. We may also form a hologram at one wavelength and view it with another. The magnification is

$$
M=\left(1-\frac{\lambda_{1}}{\lambda_{2}} \frac{z_{1}}{z_{2}}-\frac{z_{1}}{z_{2}}\right)^{-1}
$$

where the $z$ 's are defined in FIGURE 8 and $\lambda_{1}$ and $\lambda_{2}$ are, respectively the wavelengths of the radiation used for forming and viewing the hologram. This expression applies to the holographic real (or conjugate) image. We may produce whatever magnification we choose by appropriate selection of the optical geometry. This method, however, is undesirable because the holographic image, when formed with non-unity magnification, has all the aberrations of a simple lens system, including spherical aberrations, coma, etc. ${ }^{13,14}$ Judicious choice of parameters can minimize these aberrations, as the experimental results show (FIGURE 9). Nonetheless, this method is not very desirable.

For best results, the magnification should be done with a microscope, either in the recording or in the reconstruction process. This kind of microscopy has

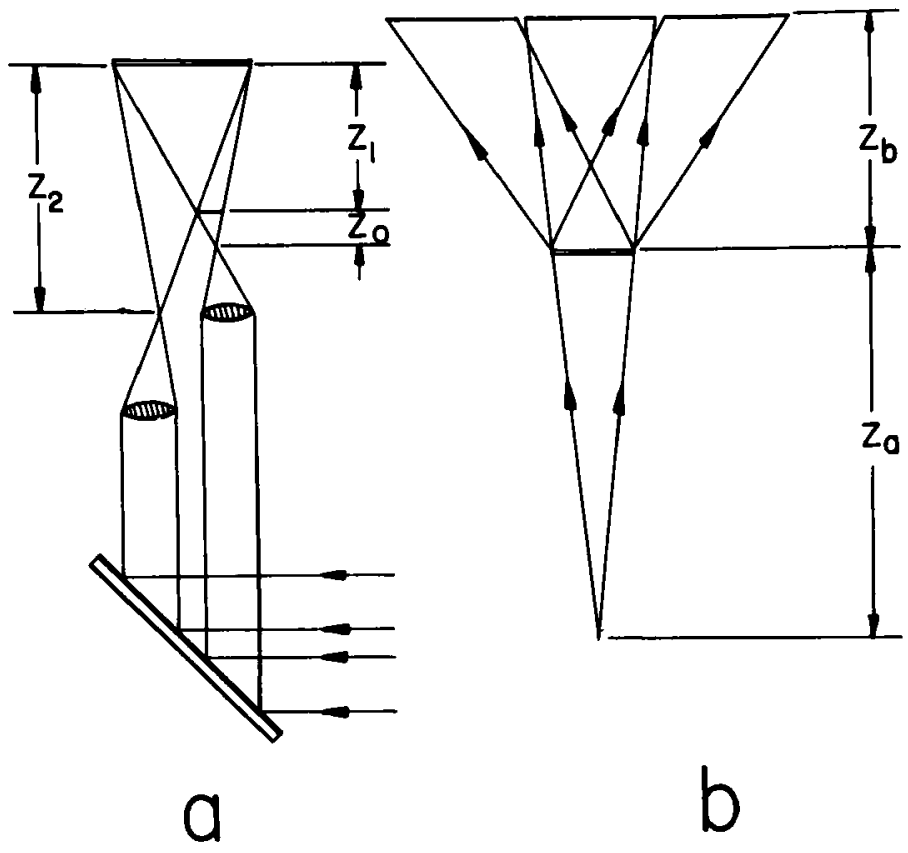

Figure 8. Magnification by holography. (a) The recording process. The object is placed a distance $Z_{0}$ from the point focus of the object beam; the hologram is formed a distance $Z_{1}$ from the object. (b) The viewing process. The holographic real image forms a distance $\mathrm{Z}_{0}$ from the hologram, in one of the two first-order diffractions. 


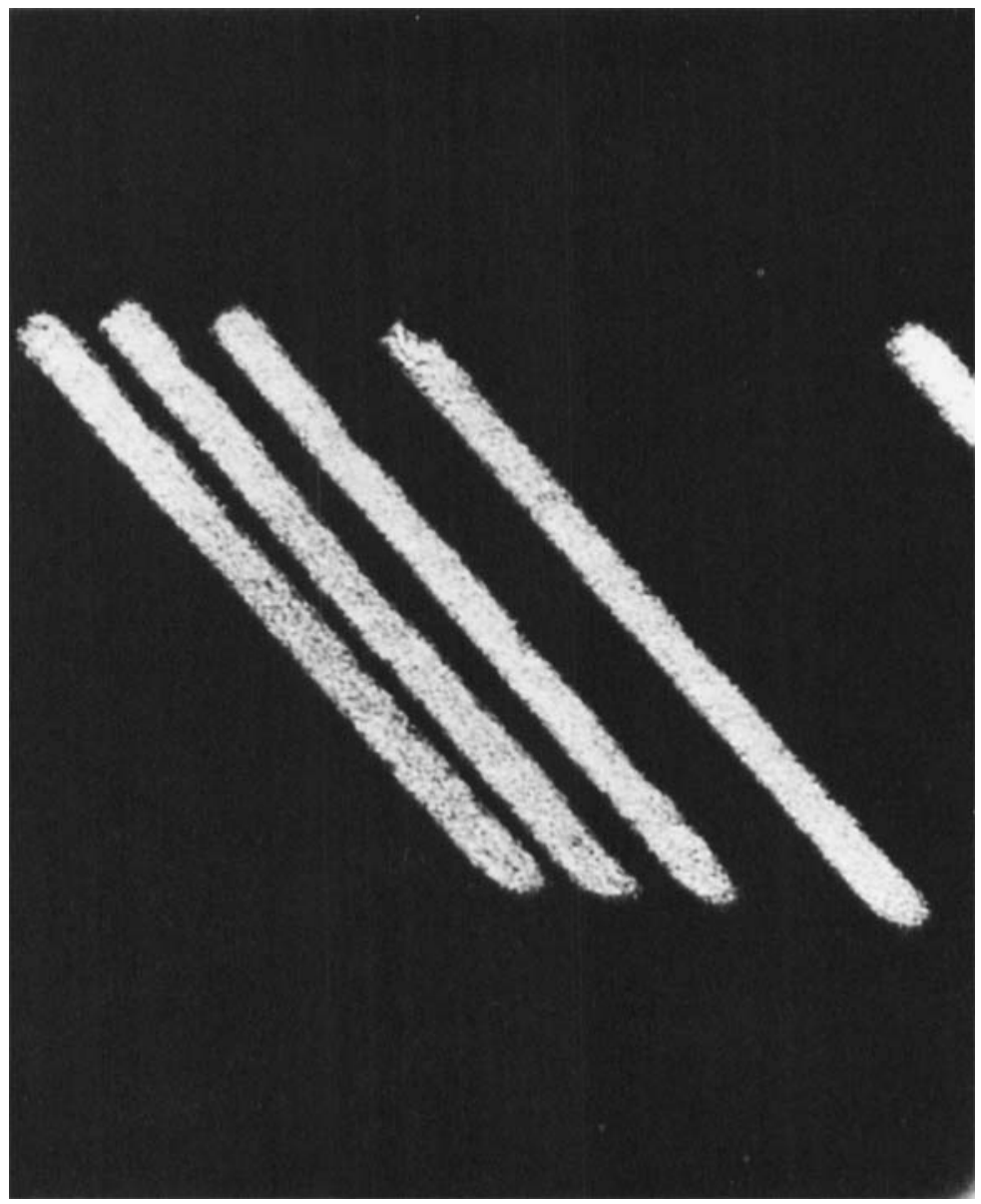

FIGURE 9. A bar pattern magnified $130 \times$ by the holographic process. Resolution is estimated to be about 3 microns.

been reported by Carter $e t$ al. ${ }^{15}$ and by van Ligten. ${ }^{16}$ Holomicrography, as demonstrated by van Ligten's recent results, appears promising.

The holographic record reproduces the recorded wave complete with amplitude and phase. Consequently, the holographically produced image can be substituted for the original object in a wide variety of situations, as noted and demonstrated by Horman. ${ }^{17}$ For example, we may perform phase contrast, Schlieren, or interference techniques on the holographic image, just as we could do on the original object. Further, spatial filtering for pattern recognition as described in the previous section, also can be performed using the hologram instead of the specimen.

Even more significant is the observation that the holographic image can be interfered with the original object, or two successively recorded exposures on the same photographic record. ${ }^{18-21}$ This method of interference has been called holographic interferometry. It can be used to determine minute changes in the 
Annals New York Academy of Sciences

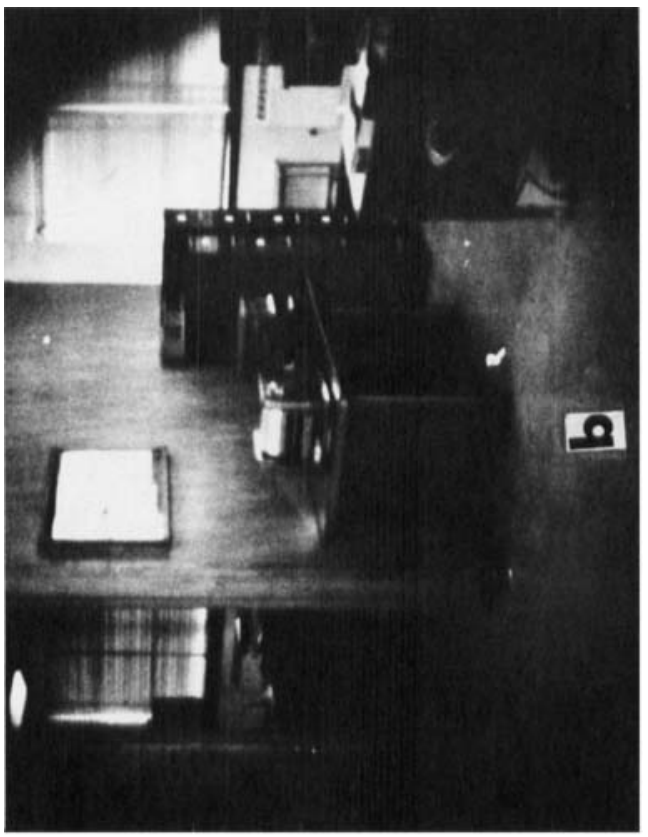

$\stackrel{\dot{c}}{\mathbf{c}}$

อ

4

을

氙

范

胥

s

․․ㅇ

용

营

总

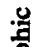

高

용

$\sigma$

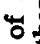

色象

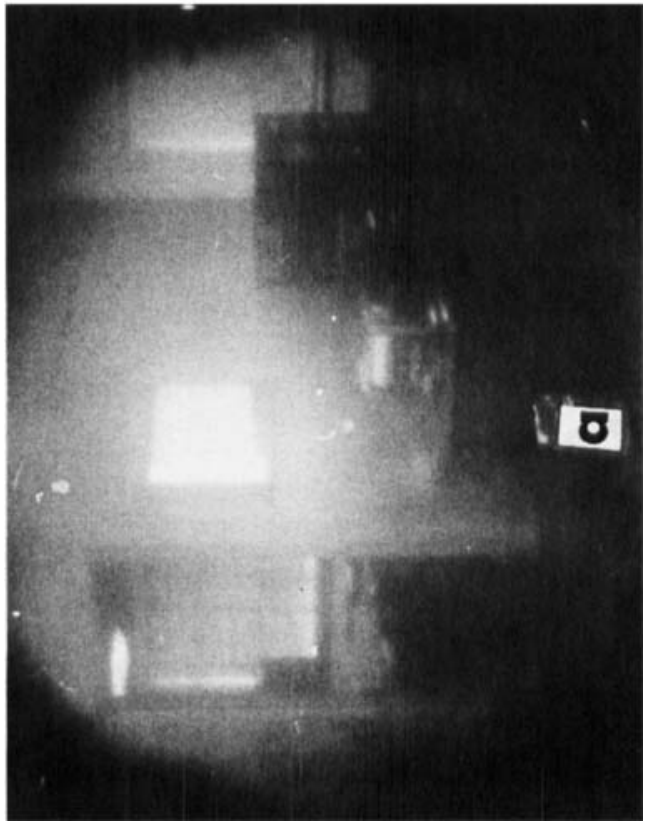

过

文壳

8.

들

블

웅

8

古

空

四

졸

형

동

湿

$\infty$

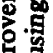

品

自

$\circ$

g.

苛

的 
object over the period of time between the successive exposures or between the time the hologram is made and the time at which it is interferometrically compared with the original object. Any change in the shape of the object, in its refractive index, or in any other property that alters the optical path of the light that interrogates the object, causes interference fringes that enable these changes to be accurately measured.

Holograms can be readily made from prepared slides, using either continuous wave or pulsed lasers. Specimens can also be holographed in vivo using a pulsed laser which delivers its energy in a time duration so short that the object motion is negligible. Without the pulsed laser, holography of live specimens or of other moving objects hardly would be possible since holography is a type of interferometry, and interferometry requires that the object be stationary to within a fraction of a light wavelength during the exposure.

Our experience with pulsed lasers has been limited; Brooks et al. ${ }^{19}$ and Thompson and Parrent, ${ }^{22}$ however, have demonstrated impressively the potential of pulsed laser holography.

The final topic of interest here is the use of holograms as optical elements. A hologram can be made of a wavefront of arbitrary shape, either spherical or aspherical. The resulting hologram then reshapes any wavefront that traverses it, just as a lens reshapes an incident wavefront. If a hologram is made of a spherical wavefront, the hologram becomes, in effect, a spherical lens. It would, in fact, be a Fresnel zone plate or diffraction lens, the behavior of which is similar to that of a conventional lens. A hologram of an aspherical wavefront becomes an aspherical optical element. Thus, by holographic means we can produce an aspheric element of any conceivable figure simply by generating a corresponding wavefront. Alternatively, the appropriate hologram can be generated by a digital computer.

Such an optical element has been used to correct the spherical aberration of a lens. This is accomplished by holographically recording the aberrant wavefront from a lens illuminated by a monochromatic point source. In the recording process, the complex conjugate of the wave is generated and becomes the real image term. The real image wave for the hologram thus has an aberration conjugate to that of the lens, and the two elements (hologram and lens) in tandem produce an image free from spherical aberration. An example is shown in FIGURE 10.

\section{Conclusions}

Coherent optical techniques find increasing use in data processing; these techniques are particularly attractive when the data in inherently two dimensional and if the data already exists in optical form as, for example, in the case of photographic transparencies. The operations of autocorrelation, convolution, matched filtering, or indeed almost any linear operation, are readily performed optically, either as a two-dimensional operation or as a multiplicity of one-dimensional operations. In addition, holographic techniques such as holomicroscopy and interferometric holography show promise in biological applications.

\section{References}

1. Maréchal, A. \& P. Croce. 1953. C. R. Acad. Sci. 237: 607.

2. Rhodes, JR., J. E. 1953. Am. J. Phys. $21: 227$.

3. O'NeILL, E. L. 1956. IRE Trans. Inform. Theory IT-2: 56.

4. Cutrona, L. J., E. N. Leith, C. J. Palermo \& L. J. Porcello. 1960. IRE Trans. Inform. Theory IT-6: 386 . 
5. Maréchal, A. \& M. Francon. 1960. Diffraction structure des images. : Revue d'Optique.

6. VANDER LugT, A. 1964. IEEE Trans. Inform. Theory IT-10: 139.

7. Vander Lugt, A., F. B. Rotz \& A. Klooster, JR. 1965. Optical and Electro-Optical Information Processing. Ed. J. T. Tippett, et al. MIT Press, Chap. 7: 125.

8. VANDer Lugt, A. 1966. Proc. IEEE 54: 1055.

9. TURIN, G. L. 1960. IRE Trans. Inform. Theory IT-6: 311.

10. Gabor, D. 1949. Proc. Roy. Soc. (Lond.) A 197: 454.

11. Leith, E. N. \& J. Upatnieks. 1964. J. Opt. Soc. Am. 54: 1295.

12. El-Sum, H. M. A. \& A. V. Baez. 1955. Phys. Rev. 99: 624.

13. Meier, R. 1965. J. Opt. Soc. Am. 55: 987.

14. Armstrong, J. 1965. IBM, J. Res. Develop. 9: 171.

15. CarTer, W., et al. 1966. J. Quantum Electron. QE2: 44.

16. VAN Ligten, R. 1967. J. Opt. Soc. Am. 57: 564.

17. Horman, M. 1965. Appl. Opt. 4: 333.

18. Powell, R. \& K. Stetson. 1965. J. Opt. Soc. Am. 55: 612, 1570, 1593.

19. Brooks, R., L. Heflinger \& R. Wuerker. 1965. Appl. Phys. Letters 7: 92, 248.

20. Collier, R., J. Doherty \& K. Pennington. 1965. Appl. Phys. Letters 7: 223.

21. Burch, J. 1965. Viscount Nuffield Memorial Paper, Inst. of Prod. Engineers, Royal Aeron. Soc.

22. Thompson, B., J. Ward, W. Zinky. 1965. J. Opt. Soc. Am. 55: 1566. 